\title{
Supporting Information: Phase Transition in a Heterogeneous Membrane: Atomically Detailed Picture
}

Arman Fathizadeh, ${ }^{1}$ Mason Valentine, ${ }^{2}$ Carlos R. Baiz, ${ }^{2}$ Ron Elber,,${ }^{1,2}$

${ }^{1}$ Oden Institute for Computational Science and Engineering, and ${ }^{2}$ Department of Chemistry, University of Texas at Austin, Austin, Texas, 78712

\section{Simulation set up}

A mixture of DPPC and DLPC bilayer with ratio of 1:1 on both leaflets was constructed using CHARMM-GUI ${ }^{1}$ with lipids distributed randomly in the membrane. The system contains 100 phospholipids on each leaflet. The bilayer was solvated with TIP3P water molecules and salt concentration of $0.15 \mathrm{M}$ sodium chloride $(\mathrm{NaCl})$. The solvated system consists of approximately 46,000 atoms. Periodic boundary conditions were applied in all directions. All the simulations have been conducted with the NAMD program ${ }^{2}$ and CHARMM36 force field. ${ }^{3}$ The system were minimized using conjugate gradient algorithm for 10000 steps and then equilibrated in the NPT ensemble using Nose-Hoover Langevin piston pressure control ${ }^{4,5}$ for $20 \mathrm{~ns}$ at pressure of $1 \mathrm{~atm}$ and three different temperatures of $283 \mathrm{~K}, 303 \mathrm{~K}$, and $340 \mathrm{~K}$. Then for each temperature, system was equilibrated in the NVT ensemble for 10ns using a Langevin thermostat. The water molecules were kept rigid using the SETTLE algorithm ${ }^{6}$ and all other bond lengths with hydrogen atoms were kept fixed with the SHAKE algorithm. ${ }^{7}$ The time step is $1 \mathrm{fs}$ in all the simulations. A real space cutoff distance of $12 \AA$ was used and Particle Meshed Ewald (PME) ${ }^{8}$ accounted for long range electrostatic calculations.

\subsection{MDAS}

Recently, the method of MDAS (Molecular Dynamics with Alchemical Step) was introduced for equilibration of lipid mixtures. ${ }^{9}$ This method is a concrete implementation of the general 
formulation of non-equilibrium candidate Monte Carlo move. ${ }^{10}$ Because of the slow lateral diffusion of the lipids in the membranes, several microseconds to milliseconds of conventional MD simulation are required to reach equilibrium. Using the MDAS algorithm enables us to perform trial moves and switch between different lipid types to enhance the sampling of the lipid mixtures. We have illustrated that speed-up by a factor of $\sim 1,000$ can be obtained.

Every MDAS move consists of two parts that we refer to as a D-trajectory and an M-trajectory. The M-trajectory is the exchange event where the alchemical step occurs, and the D-trajectory is an unbiased MD trajectory between two exchange events. Consider a dense system of $N$ particles with spatial positions denoted by coordinate vectors $\boldsymbol{r}_{\boldsymbol{i}}$. We are seeking an exchange move between two sub-groups of the system e.g. group $i$ of type $k$ and group $j$ of type $l, l \neq k$. An instantaneous exchange Monte Carlo move, will change the potential energy of the system from $U\left(r_{1}, \ldots, r_{i}^{k}, \ldots, r_{j}^{l}, \ldots, r_{N}\right)$ to $U\left(r_{1}, \ldots, r_{j}^{l}, \ldots, r_{i}^{k}, \ldots, r_{N}\right)$. In a dense system like a phospholipid membrane such a move has a very small chance for acceptance due to van der Waals clashes. Therefore, we a seek a gradual sequence of moves that lead to the same exact ensemble but with a significantly higher chance of acceptance.

An M-trajectory can be parametrized with a parameter $\lambda$ such that when $\lambda=0$ the system is before the exchange and at $\lambda=1$ the move is complete. The $\lambda$ pathway from $\lambda=0$ to $\lambda=1$ is not unique and can be optimized by the user by (for example), minimizing the work. According to Jarzynski, ${ }^{11}$ the work done on the system during an $M$ trajectory can be written as:

$$
w=\int_{t=0, \lambda(0)=0}^{t^{\prime}, \lambda\left(t^{\prime}\right)=1} \frac{\partial U_{\lambda}}{\partial \lambda} \frac{d \lambda}{d t} d t
$$

Where $U_{\lambda}$ is the potential energy of the system at a particular $\lambda$ and the time $\mathrm{t}$ parametrizes the path. The M-trajectories are conducted with the potential $U_{\lambda}$ for a short time, $\Delta t$, and then $\lambda$ is adjusted by a small increment to give $\lambda+\Delta \lambda$ and a new potential $U_{\lambda+\Delta \lambda}$. The increments in $\lambda$ starts from $\lambda=0$ continues until $\lambda=1$ is reached. Each increment contributes to the total work as written in Eq. (2):

$$
w=\sum_{i=1}^{L} w_{i}=\sum_{i=1}^{L}\left[U_{\lambda i+\Delta \lambda}\left(\boldsymbol{r}_{i}\right)-U_{\lambda i}\left(\boldsymbol{r}_{i}\right)\right]
$$


Where $L=\frac{1}{\Delta \lambda}$. Note that both potentials are calculated at the same coordinate at time $t_{i}$. It is the last time in which the potential was $U_{\lambda_{i}}$ and the first time it was $U_{\lambda_{i}+\Delta \lambda}$. The weight of the M trajectory, which is the trial MC move is $e^{-\beta w}$. The final configuration of the trajectory will be accepted/rejected in a Metropolis-like criterion:

$$
P_{\text {acceptance }}=\min \left[1, e^{-\beta w}\right]
$$

In our recent paper ${ }^{9}$ we have used MDAS for efficient mixing of DOPC:POPC mixtures. DOPC:POPC are well mixed in the fluid phase for a broad range. Here, we are using it to conduct exchange moves between DPPC and DLPC molecules.

The MDAS algorithm for this system was implemented following these steps:

1- Two lipid molecules, each of a different type, are randomly selected from the same leaflet.

2- Both lipids are being replaced by a mixed topology (Fig S1A) which contains all the atoms that are present in both DPPC and DLPC.

3- An M-trajectory gradually turns the dual topology molecule located at the position of DPPC into DLPC and vice versa. The Van der Waals and electrostatic interactions are gradually scaled using a parameter $\lambda$ that parametrizes the M-trajectory. In the current study, $\lambda$, changes by a small increment of $d \lambda=0.00005$ and the system is propagated for 80 steps at the new potential before applying the next $\lambda$ increment (Fig S1B). The work increment at each step is calculated. A linear path was selected to evolve the electrostatic interactions but for the Lennard-Jones interactions we applied a soft-core potential to avoid the so-called end point catastrophes ${ }^{12}$ :

$$
U_{s o f t}=4 \varepsilon \lambda_{L J}\left[\left(\frac{\sigma_{i j}}{r_{i j}^{2}+\eta\left(1-\lambda_{L J}\right)}\right)^{6}-\left(\frac{\sigma_{i j}}{r_{i j}^{2}+\eta\left(1-\lambda_{L J}\right)}\right)^{3}\right]
$$

Where $\eta=5.0$ and $\lambda_{L J}$ changes from 0 to 1 . 
4- The total work is calculated from summation of the work increments and the move is accepted or rejected according to Eq. (3).

5- If the move gets rejected, we go back to step 1. If the move gets accepted, we switch the lipids, reverse the direction of the velocities of all particles, and perform an unbiased MD (D-trajectory) for 500 picoseconds in NPT ensemble to enable the membrane to adjust its dimensions.

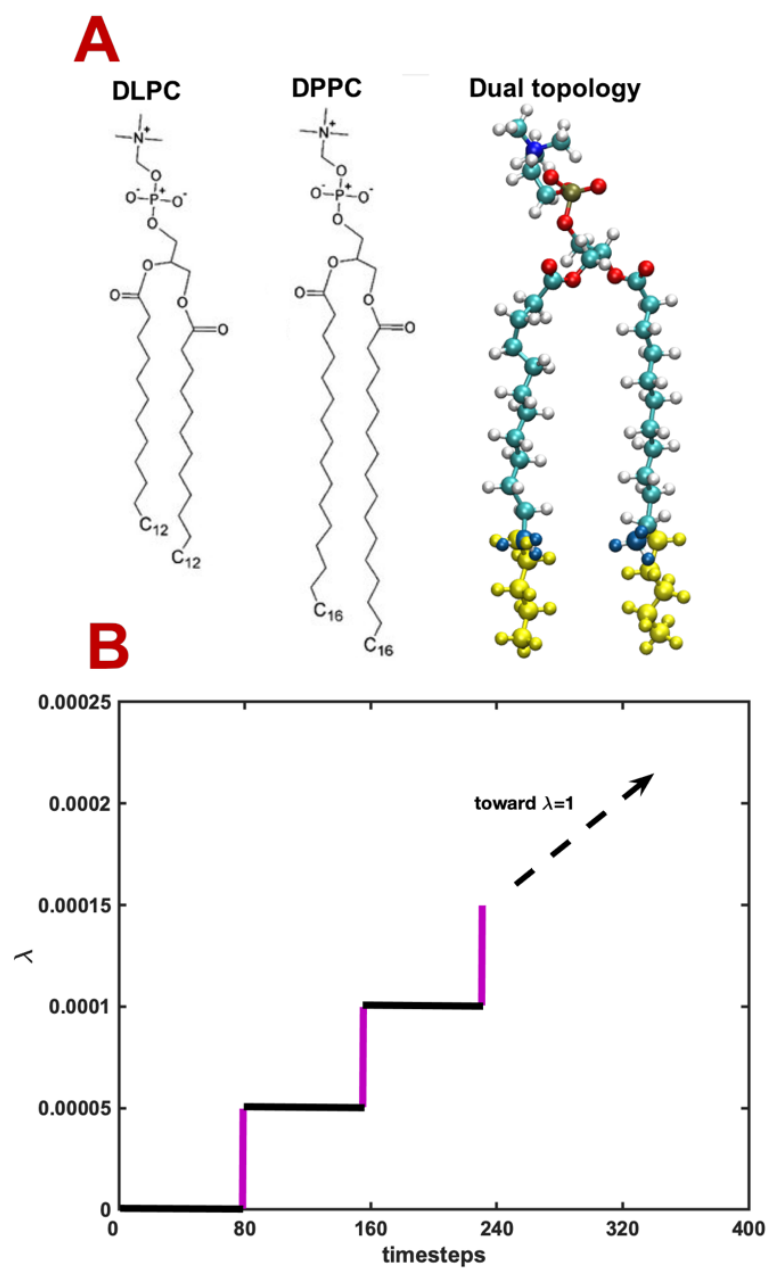

Fig S1. Panel A: The schematic representation of the DLPC and DPPC lipid molecules with the dual topology containing all the atoms required for MDAS calculations for the switch between the two lipids. Panel B: The evolution of the parameters during an M trajectory used in the 
current study. The work is computed by a summation over the work increments on the purple lines.

\section{Simulation results}

\subsection{The number of neighbors:}

To quantify the differences between the phases over MDAS simulations we define the index (I) as the average number of neighbors within the same leaflet for every DPPC (or DLPC) that are from the same type. The "neighbor" is defined in a similar way as in reference [13] by dividing the $2 \mathrm{D}$ surface of the membrane into Voronoi cells using the $\mathrm{C} 2$ atom of every lipid as the representative for the lipid. An example of this tessellation of the 2D space is shown in Fig. S2. The to,e evolution of the indices I of different runs is shown in Fig. S3.

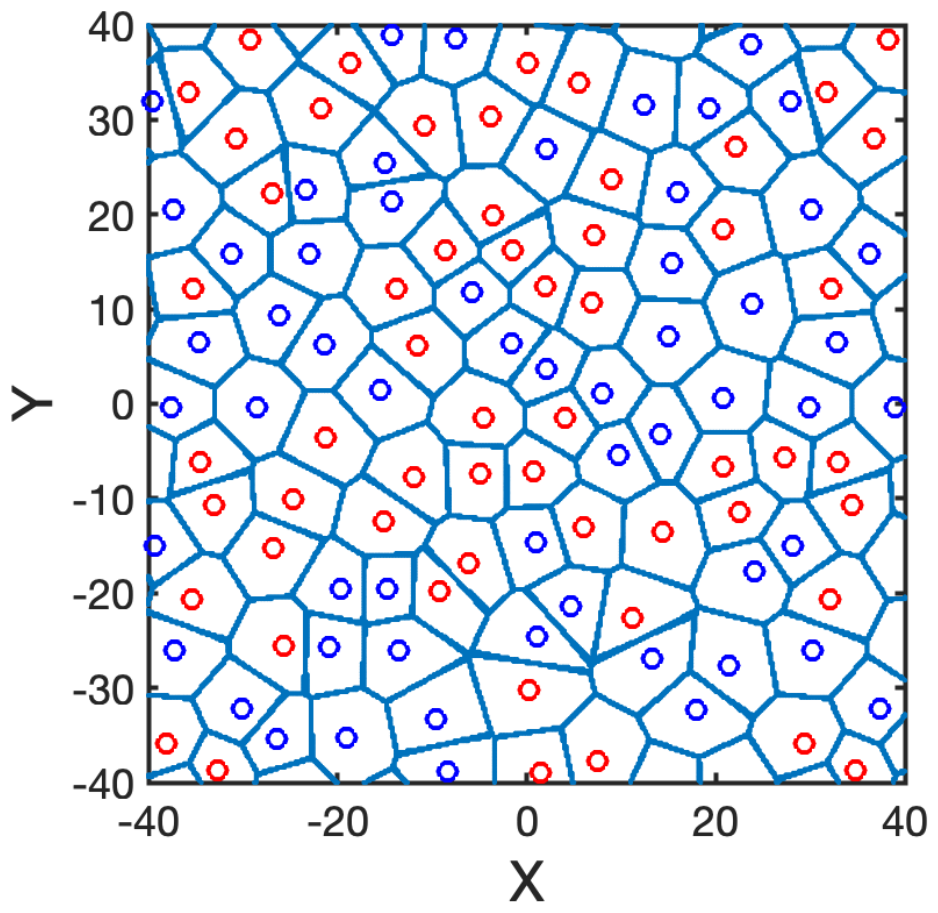

Fig S2- Example of Voronoi tessellation of the membrane in XY plane to define the neighboring lipids. The red and blue circles represent $\mathrm{C} 2$ atoms of DPPC and DLPC 
respectively. Two lipids are considered to be neighbors if they have a shared boundary of their corresponding Voronoi cells.
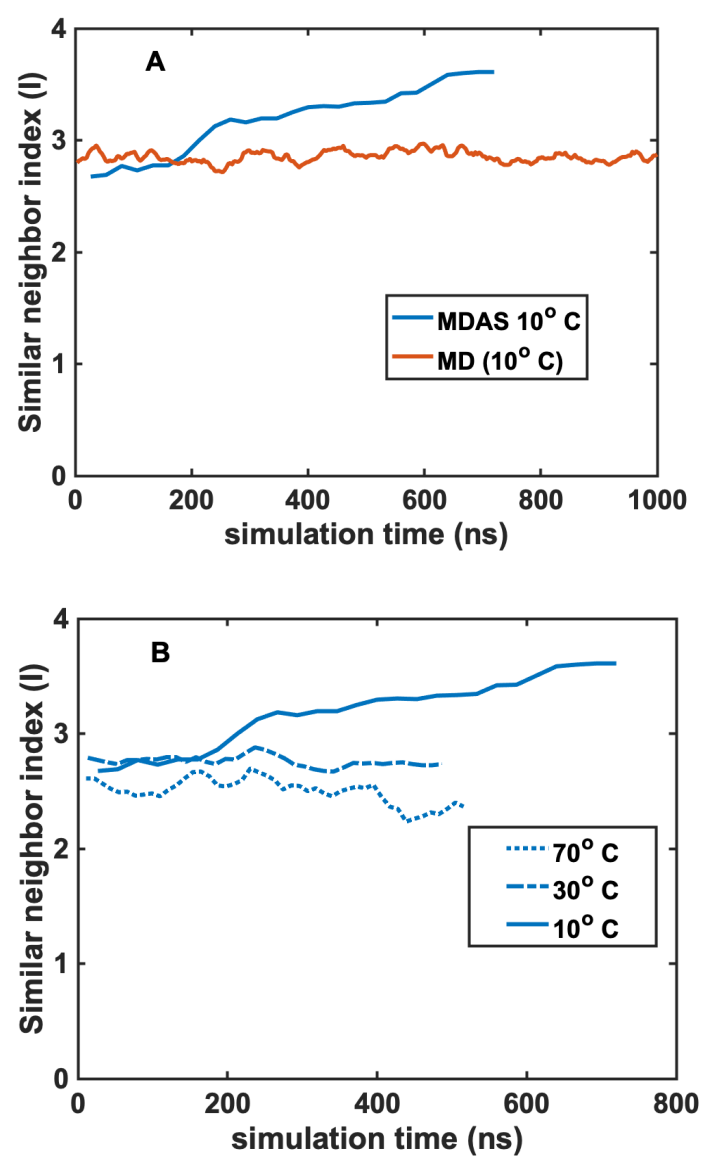

Fig S3- The evolution of the average number of neighbors during MD and MDAS simulations at $10^{\circ} \mathrm{C}$ (panel A) and MDAS simulations at different temperatures (Panel B)

In Figure S3A we illustrate that MDAS is on a clear path for cluster growth in agreement with experiment (see main text). The process of phase transition and phase separation are extremely slow and are difficult to complete even with the enhanced sampling of MDAS. Nevertheless, significant growth in the number of neighbors is already observed, which is consistent with Figure 2 of the main text. A microsecond trajectory of conventional MD did not form a cluster, presumably because the time for cluster formation is too long for conventional MD. 
In Figure S3B we compare different Milestoning simulations. In accord with our paper ${ }^{9}$ MDAS converges extremely rapidly to equilibrium in the fluid phase. Indeed, we observe that the simulations at $30^{\circ} \mathrm{C}$ and $70^{\circ} \mathrm{C}$ are reasonably stable. In contrast the simulation at the lower temperature is showing a growing number of neighbors and increasing order, which will presumably end when the cluster will contain all the DPPC molecules in a compact conformation.

\subsection{The Order Parameter $S_{C D}$ :}

The $\mathrm{S}_{\mathrm{CD}}$ order parameter is defined as:

$$
S_{C D}=\left(\frac{1}{2}\right)<3 \cos ^{2}(\theta)-1>
$$

where $\theta$ is the angle between a $\mathrm{C}-\mathrm{H}$ bond and the normal to the membrane as a reference vector. The brackets denote an average over all the lipids and times. Fig. S4 shows this order parameter for different carbons on the tails of the lipids, The data are provided at different temperatures and for the pure and mixed membranes. 


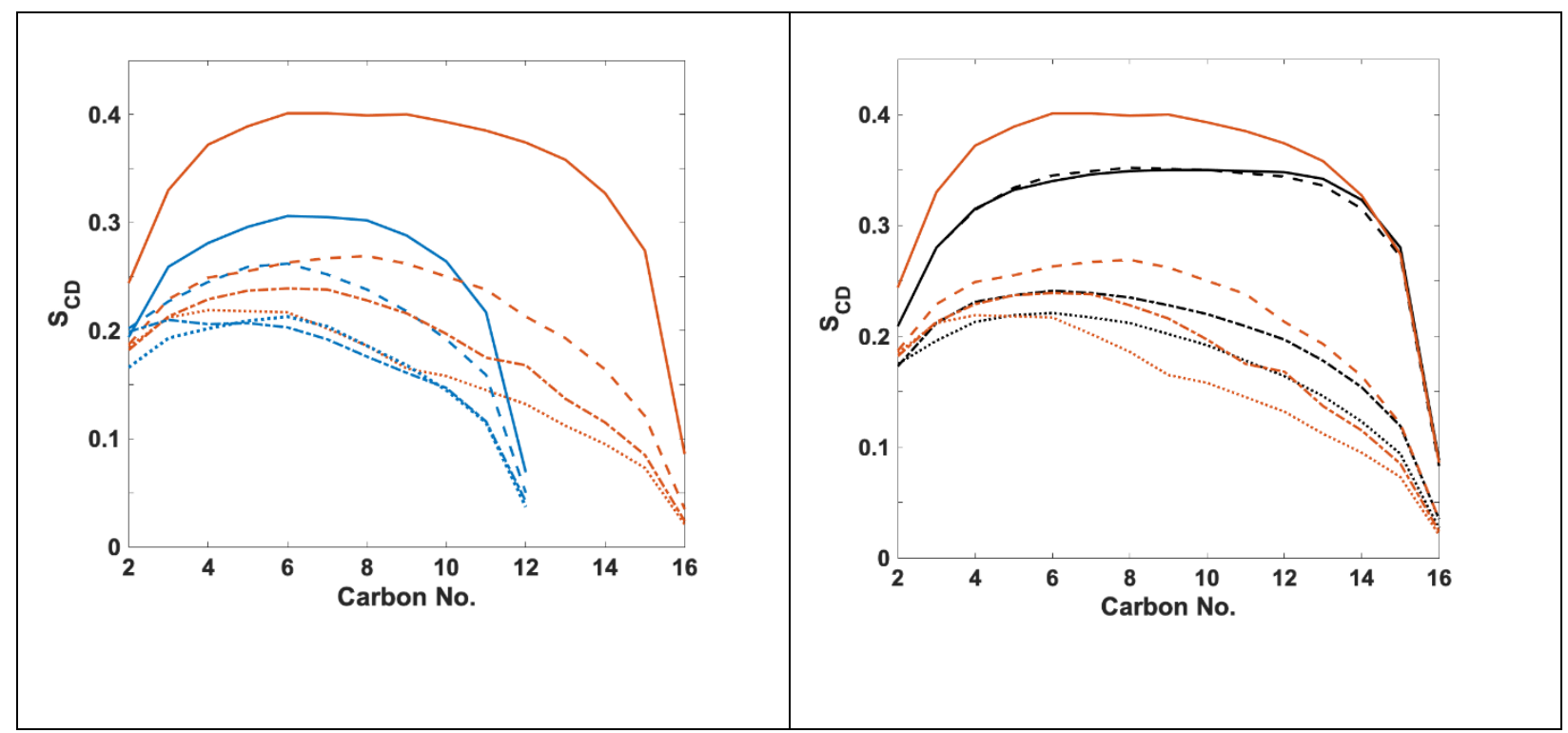

Fig S4- The order parameter $\mathrm{S}_{\mathrm{CD}}$ as a function of the index of the lipid carbons. The left panel shows the order parameters in the 1:1 mixture, DPPC is in red and DLPC in blue. The right panel shows only DPPC where the red line is the DPPC in the mixture (the same as the left panel) and the black line is for the pure DPPC membrane. In both panels, the solid line is at temperature of $10^{\circ} \mathrm{C}$, dashed line at $30^{\circ} \mathrm{C}$, dashed-dotted line for $50^{\circ} \mathrm{Cand}$ dotted line at $70^{\circ} \mathrm{C}$. Note that the order parameter of DPPC is smaller in the pure versus the mixed phase. This is explained inn the main text (Figure 4) showing that in the pure phase DPPC is more tilted compared to the mixture.

\section{Synthesis and Sample Preparation}

Isotope-labeled dipalmitoyl phosphatidylcholine (DPPC $\left.\left({ }^{13} \mathrm{C}\right)\right)$ was synthesized via Steglich esterification following a procedure developed by Ichihara et al. ${ }^{14}$ Briefly, $0.1 \mathrm{mmol}$ sn-glycero3-phosphocholine (GPC) was dissolved in methanol and adsorbed onto Celite. The solvent was evaporated from the Celite in a vacuum desiccator for 2 hours and then the solids were dried in a lyophilizer overnight to remove water. The GPC and Celite were combined with 2 equivalents 1${ }^{13} \mathrm{C}$ palmitic acid, purchased from Cambridge Isotope Labs, and 2.5 equivalents 4dimethylaminopyridine (DMAP) in a $10 \mathrm{~mL}$ round-bottom flask. The solids were suspended in anhydrous dichloromethane under nitrogen and 5 equivalents of $\mathrm{N}$-(3-dimethylaminopropyl)-N'ethylcarbodiimide hydrochloride $(\mathrm{EDC} \cdot \mathrm{HCl})$ were added. The suspension was stirred under nitrogen for 24 hours, then filtered to remove Celite. The filtrate was dissolved in chloroform and 
rinsed four times with $0.1 \mathrm{M} \mathrm{HCl}$ in 50:50 methanol:water to remove DMAP and the hydrolyzed EDC $\cdot H C l$. Finally, DPPC $\left({ }^{13} \mathrm{C}\right)$ was purified via a silica flash column with 65:25:4 $\mathrm{CHCl}_{3}: \mathrm{MeOH}: \mathrm{H}_{2} \mathrm{O} \mathrm{v}: \mathrm{v}: \mathrm{v}$ as the eluent. The identity and purity of the product were confirmed using NMR and FTIR spectroscopy, and then a stock solution was prepared by dissolving a known mass of the product in chloroform.

A solution of dilauroyl phosphatidylcholine (DLPC) dissolved in chloroform was purchased from Avanti Polar Lipids and used without further purification. Lipids solutions were transferred to vials, and then the solvent was removed under a dry stream of nitrogen, followed by vacuum for at least 2 hours. A solution of $150 \mathrm{mM} \mathrm{NaCl}$ in deuterated water was then added to hydrate and suspend the lipids. To homogenize the samples and reduce scatter, 6 freeze thaw cycles were performed followed by ultrasonication in a bath sonicator for 20 minutes prior to IR measurements.

\subsection{Infrared Spectroscopy}

FTIR spectra were recorded on a Bruker Vertex 70 spectrometer. Samples were held between two calcium fluoride windows separated by a $50 \mu \mathrm{m}$ thick Teflon spacer in a homemade brass sample cell, and a bath circulator was used to heat and cool the brass sample cell. Phase transition temperatures were measured by measuring spectra every $2^{\circ} \mathrm{C}$, starting at $4^{\circ} \mathrm{C}$ and ending at $44^{\circ} \mathrm{C}$. Once the sample cell reached a set temperature, it was held at that temperature for five minutes to allow the sample to equilibrate prior to the measurement of the spectrum. In order to minimize the absorption bands from water vapor in the air, we continuously purged the spectrometer with dry air. Temperature-dependent FTIR spectra were recorded from 400 to $4000 \mathrm{~cm}^{-1}$ and the ester carbonyl $\left(\sim 1730 \mathrm{~cm}^{-1}\right)$ and symmetric $\mathrm{CH}_{2}$ stretching $\left(2850 \mathrm{~cm}^{-1}\right)$ bands were analyzed. 


\section{Characterization of DPPC $\left({ }^{13} \mathrm{C}\right)$}

1H NMR: (400 MHz, chloroform-d) $\delta=5.24-5.16(\mathrm{~m}, 1 \mathrm{H}), 4.43-4.35(\mathrm{dt}, 1 \mathrm{H}), 4.34-4.25(\mathrm{~m}, 2 \mathrm{H})$, 4.17-4.07 (m, 1H), 3.99-3.86 (m, 2H), 3.81-3.73 (s, 2H), 3.41-3.25 (s, 9H), 2.34-2.22 (quint, 4H), $1.63-1.51(\mathrm{~m}, 4 \mathrm{H}), 1.33-1.19(\mathrm{~m}, 48 \mathrm{H}), 0.91-0.84(\mathrm{t}, 6 \mathrm{H})$

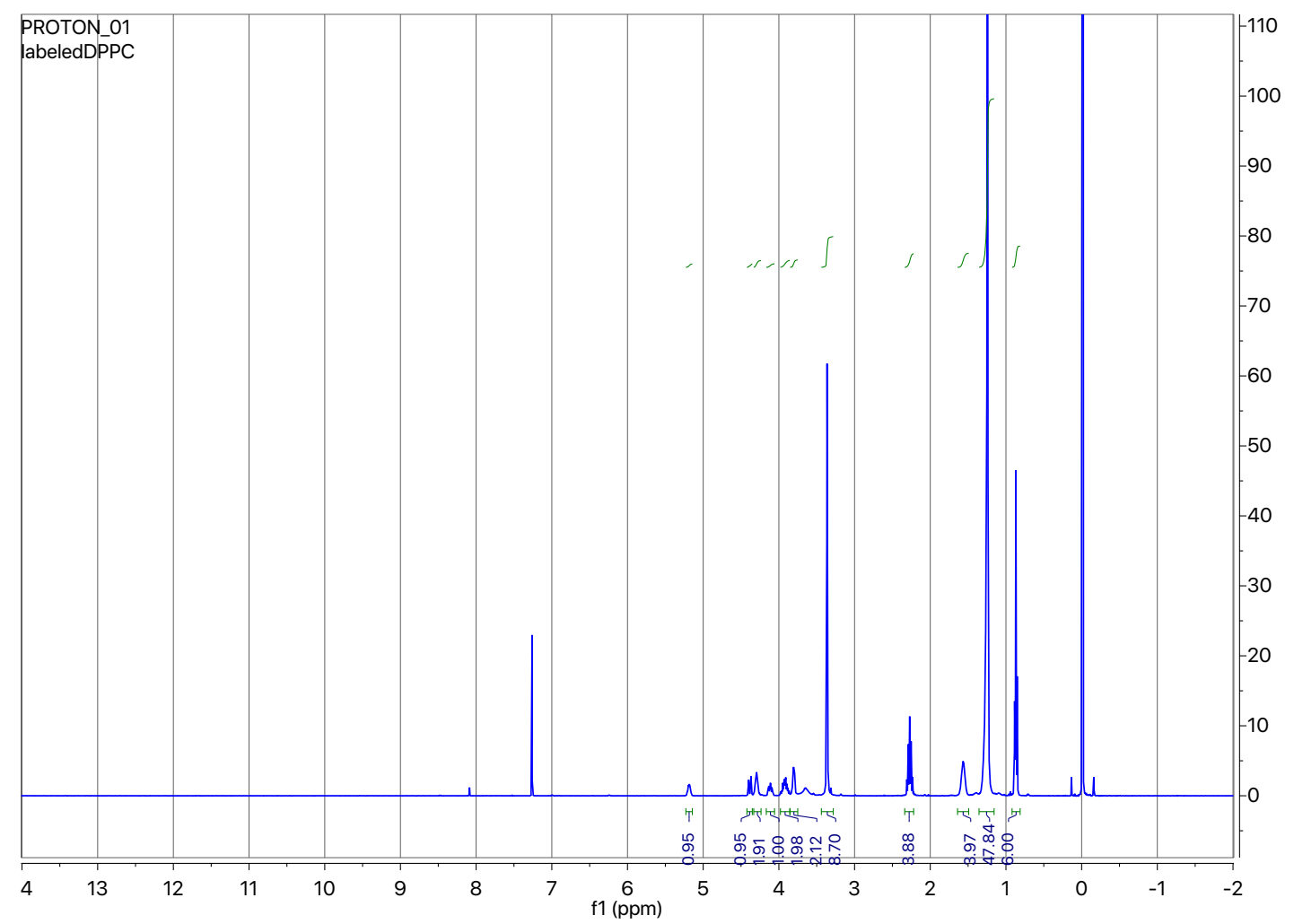

Fig S5. ${ }^{1} \mathrm{H}$ NMR spectrum of DPPC $\left({ }^{13} \mathrm{C}\right)$ in $\mathrm{CDCl}_{3}$

FTIR: vmax/cm-1 2920 and 2850 (CH2), 1695 (13COOR), 1468 (CH2), 1260 (OPO4) 


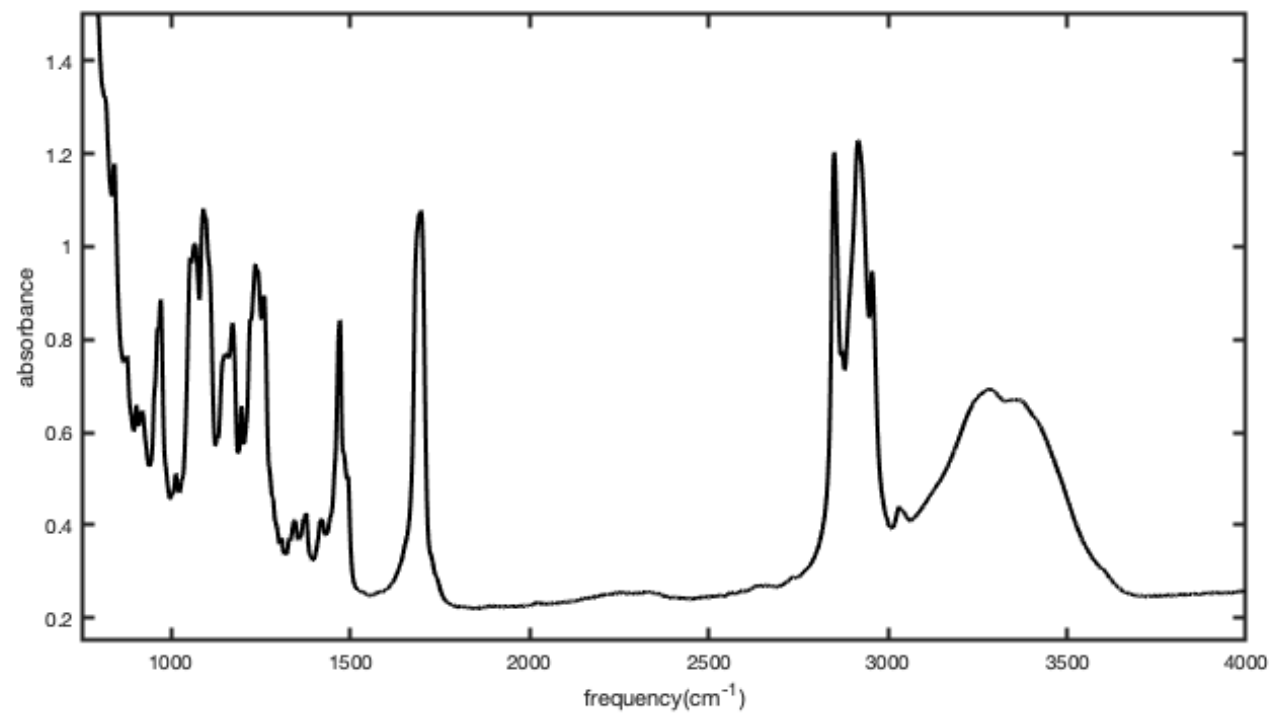

Fig S6. FTIR spectrum of DPPC $\left({ }^{13} \mathrm{C}\right)$ film dried on a $\mathrm{CaF}_{2}$ window

\section{4. $\mathrm{CH}_{2}$ Infrared Spectra}

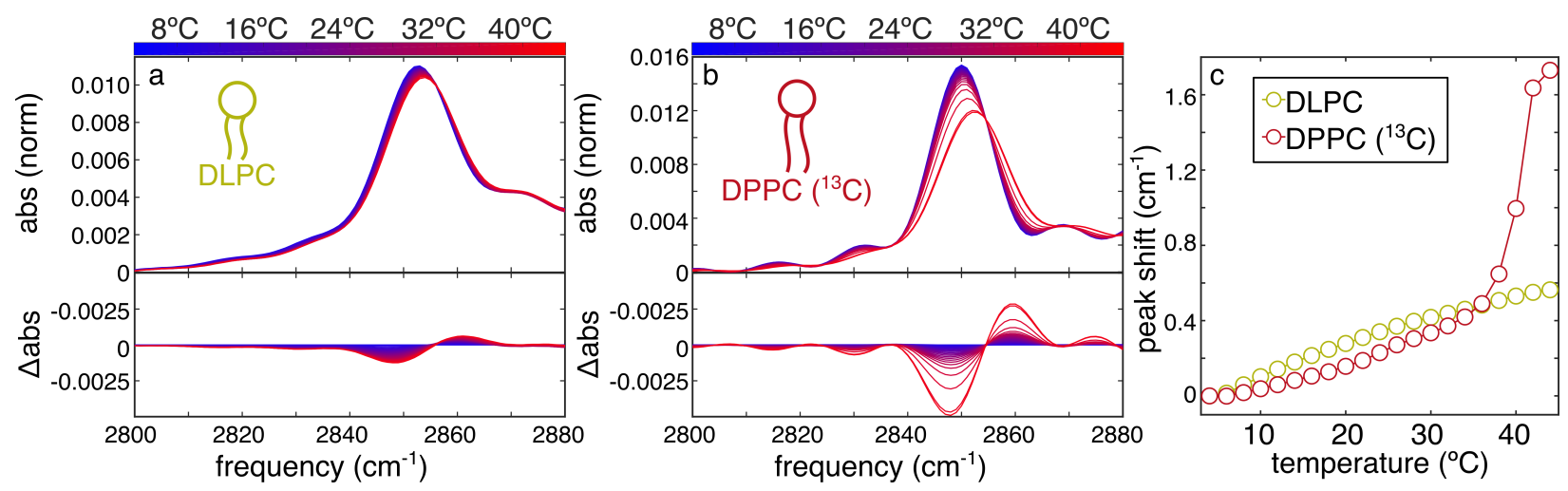

Fig S7. Temperature-dependent infrared spectra of the $\mathrm{CH}_{2}$ symmetric stretch of lipid suspensions composed of (a) DLPC and (b) DPPC $\left({ }^{13} \mathrm{C}\right)$ in $\mathrm{D}_{2} \mathrm{O}$. Absorbance spectra are shown in the upper panels, and difference spectra, calculated by subtracting the spectrum measured at $4^{\circ} \mathrm{C}$, are shown in the lower panels. (c) shifts of the peak frequency as a function of temperature, showing a discontinuous change for DPPC $\left({ }^{13} \mathrm{C}\right.$ near $\left.42^{\circ} \mathrm{C}\right)$. 

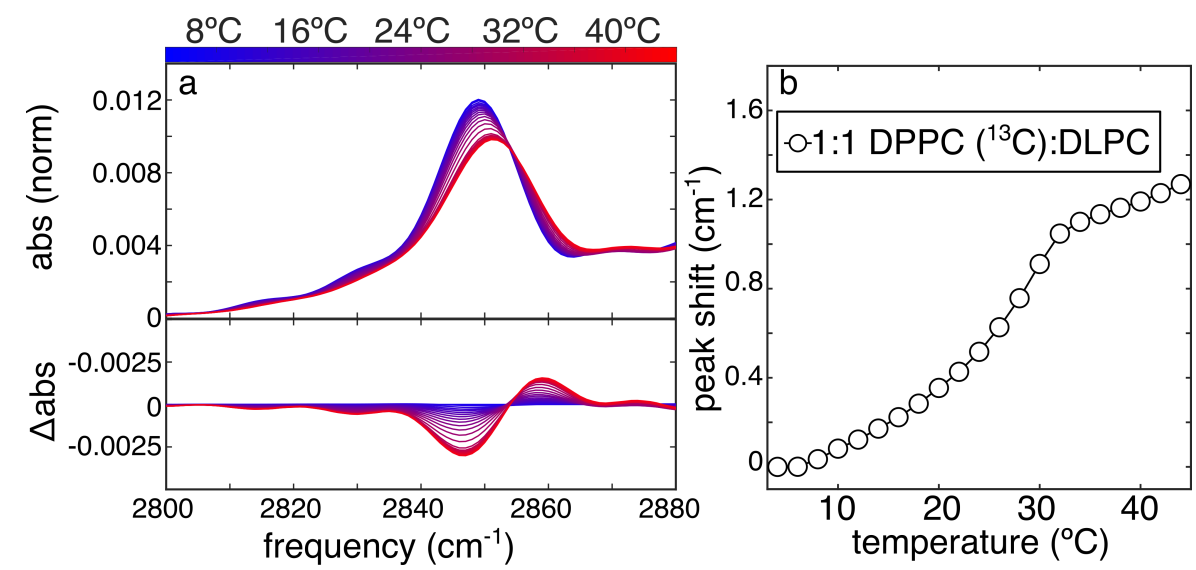

Fig S8. (a) Temperature-dependent infrared spectra of the $\mathrm{CH}_{2}$ symmetric stretch of a lipid suspension composed of a 1:1 mixture of DLPC DPPC $\left({ }^{13} \mathrm{C}\right)$ in $\mathrm{D}_{2} \mathrm{O}$. Absorbance spectra are shown in the upper panel, and difference spectra, calculated by subtracting the spectrum measured at $4^{\circ} \mathrm{C}$, are shown in the lower panel. (b) shifts of the peak frequency as a function of temperature. 


\section{References}

1. Jo, S., Lim J.B., Klauda J.B. and Wonpil I., CHARMM-GUI Membrane Builder for Mixed Bilayers and Its Application to Yeast Membranes. Biophys. J., 2009. 97(1): p. 50-58.

2. Phillips, J.C., Braun, R., Wang, W., Gumbart, J., Tajkhorshid, E., Villa, E., Chipot, C., Skeel, R.D., Kale, L., Schulten K., Scalable Molecular Dynamics With NAMD. J. Comput. Chem., 2005. 26(16): p. 1781-1802.

3. Klauda, J.B., Venable, R.M., Freites, J.A., O'Connor, J.W., Tobias, D.J., MondragonRamirez C., Vorobyov, I., MacKerell, A.D., Pastor, R.W., Update of the CHARMM All-Atom Additive Force Field for Lipids: Validation on Six Lipid Types. J. Phys. Chem. B, 2010. 114(23): p. 7830-7843.

4. Feller, S.E., Zhang, Y.H., Pastor, R.W., Brooks, B.R., Constant Pressure Molecular Dynamics Simulation: The Langevin Piston Method. J. Chem Phys., 1995. 103(11): p. 4613-4621.

5. Martyna, G.J., D.J. Tobias, and M.L. Klein, Constant Pressure Molecular Dynamics Algorithms. J. Chem. Phys., 1994. 101(5): p. 4177-4189.

6. Miyamoto, S. and P.A. Kollman, Settle: An analytical version of the SHAKE and RATTLE algorithm for rigid water models. J. Comp. Chem., 1992. 13(8): p. 952-962.

7. Ryckaert, J.-P., G. Ciccotti, and H.J. Berendsen, Numerical Integration of the Cartesian Equations of Motion of a System With Constraints: Molecular Dynamics Of N-Alkanes. J. Comp. Phys., 1977. 23(3): p. 327-341.

8. Essmann, U., Perera L., Berkowitz, M.L., Darden, T., Lee, H., Pedersen, L.G., A Smooth Particle Mesh Ewald Method. J. Chem. Phys., 1995. 103(19): p. 8577-8593.

9. Fathizadeh, A. and R. Elber, A Mixed Alchemical and Equilibrium Dynamics to Simulate Heterogeneous Dense Fluids: Illustrations for Lennard-Jones Mixtures and Phospholipid Membranes. J. Chem. Phys., 2018. 149(7): p. 072325.

10. Nilmeier, J.P., Crooks G.E., Minh, D.L., Chodera, J.D., Nonequilibrium Candidate Monte Carlo Is an Efficient Tool For Equilibrium Simulation. Proc. Natl. Acad. Sci USA, 2011. 108(45): p. E1009-E1018.

11. Jarzynski, C., Nonequilibrium Equality For Free Energy Differences. Phys. Rev. Lett., 1997. 78(14): p. 2690-2693.

12. Beutler, T.C., Mark, A.E., Vanschaik, R.C., Gerber, P.R. Van Gunsteren W.F., , Avoiding singularities and numerical instabilities in free-energy calculations based on molecular simulations. Chem. Phys. Lett., 1994. 222(6): p. 529-539.

13. Pantelopulos, G. A., Nagai, T., Bandara, A., Panahi, A., \& Straub, J. E. Critical Size Dependence Of Domain Formation Observed In Coarse-Grained Simulations Of Bilayers Composed Of Ternary Lipid Mixtures. J. Chem. Phys., 2017, 147(9), 095101.

14. Ichihara, K. Iwasaki, H., Ueda, K., Takizawa, R., Naito, H., Tomosugi, M., Synthesis of Phospatidylcholine: An improved Method without Using the Cadmium Cholride Complex of Sn-Glycero-3-Phosphacholine. Chem. Phys. Lipids 2005, 137(1), 94-99 